\title{
„Wir sind nicht bereit, unseren Platz hier zu verlieren. Weil es unser Zuhause ist, es ist unser Leben." Interview mit Mitbegründer:innen der Monitoring Group (geführt von Judith Vey und Salome Gunsch)
}

Rajaa Al Khlefawi, Namarek Al Shallal und Annika Khan

Die Frauen, mit denen wir im Rahmen eines Interviews im Frühsommer 2020 für diesen Sammelband sprachen, sind Mitbegründerinnen der Monitoring Group, einer Gruppe von Frauen mit Fluchthintergrund und Unterstützerinnen ohne Fluchthintergrund. Die Gruppe hat sich in einer Notunterkunft in einer Turnhalle 2015 gegründet. Sie setzt sich seitdem für eine Verbesserung der Unterkunftsbedingungen von Flüchtenden und für ein externes, unabhängiges Beschwerdesystem ein. Im Juni 2018 ist auf ibre Initiative hin ein Pilotprojekt zu einem unabhängigen Beschwerdemanagement in Unterkünften für Flüchtende vom Bezirksamt von Berlin - Pankow und der Senatsverwaltung für Integration, Arbeit und Soziales gestartet. ${ }^{1}$ Die Monitoring Group hat dabei sowohl auf Bezirks-als auch auf Senatsebene konzeptionell mitgearbeitet und war auch an dessen Umsetzung beteiligt. Das Pilotprojekt verfolgt einen peer-to-peer Ansatz. Das heißt, mehrsprachige Vertrauenspersonen mit Migrationsgeschichte nehmen in vertraulichen Sprechstunden in den Unterkünften die Beschwerden der Bewohner:innen entgegen. Diese werden dann durch eine Koordinierungsstelle geklärt. Zur Zeit arbeiten sie in der Zukunftswerkstatt im Berliner Stadtteil Heinersdorf, wo sie eine Kontakt- und Beratungsstelle für Menschen mit Fluchtund Migrationsbiographien aufgebaut haben.

In dem Interview erzählen Rajaa Al Khlefawi, Namarek Al Shallal und Annika Khan von den Unterbringungsbedingungen und Problemen in der Notunterkunft, von der Anschlussunterbringung in einer Gemeinschaftsunterkunft und von ihrer Wohnungssuche in Berlin. Im zweiten Teil des Interviews berichten sie von ibrem Engagement zur Verbesserung der Unterbringungsbedingungen in der Notunterkunft und der Gründung der Berlin Monitoring Group. Sie stellen ihren spezifischen Unterstützungsansatz vor und sprechen über Schwierigkeiten, mit denen sie im Zuge ihrer Aktivitäten konfrontiert waren. Zum Abschluss

1 Vgl. https://www.berlin.de/ba-pankow/politik-und-verwaltung/beauftragte/integrat ion/themen/artikel.768022.php. 
geben sie kurz einen Einblick in die Situation von Flüchtenden während der Corona-Pandemie.

\section{Unterbringung in einer Notunterkunft in einer Turnballe}

Rajaa: Ich bin Rajaa, ich bin 52 Jahre alt und habe fünf Kinder und vier Enkel. Ich kam 2015 aus dem Irak nach Deutschland. Ich habe im Irak Abitur gemacht, aber niemals gearbeitet. Aber ich habe viel Erfahrung in meiner großen Familie sammeln können. Meine Schwiegermutter und mein Schwiegervater sind schon alt und haben eine Behinderung, sie sind pflegebedürftig - ich habe sie unterstützt. Mein Vater hatte im Irak ein kleines Büro, wohin die Leute kommen, wenn sie ein Problem haben. Wir haben uns diese Probleme angehört und Lösungen dafür gefunden.

2015 mussten wir nach Deutschland fliehen. Da mein Vater Schiit ist und die Familie meines Manns Sunnit, gab es viele Konflikte. Religiös gemischte Ehen waren und sind nicht gerne gesehen und die Familien sind großen Gefahren ausgesetzt. Die Miliz hat meinen Sohn entführt. Ich konnte ihn freikaufen, aber wir haben uns dann entschieden nach Deutschland zu fliehen. Ich arbeite jetzt in der Zukunftswerkstatt Heinersdorf. Wir haben dort viel zu tun in der Sozialberatung. Unter anderem ist ein wichtiges Arbeitsthema, dass viele Frauen von Gewalt betroffen sind.

Namarek: Ich bin Namarek, ich komme aus dem Irak und bin 29 Jahre alt. Ich habe zwei Kinder, einen elfjährigen Jungen und ein eineinhalbjähriges Mädchen. Ich kam 2015 nach Deutschland. Das erste Jahr, von 2015 bis 2016 lebten wir in einer Turnhalle. Dann wechselten wir in eine Gemeinschaftsunterkunft. Dort war es etwas, aber nicht sehr viel besser. Wir haben zwei Jahre in der Gemeinschaftsunterkunft verbracht. Im Oktober 2018 haben wir eine Wohnung gefunden. Ich habe meinen Sprachkurs bis zum Niveau B1 absolviert und mache derzeit den Führerschein.

Annika: Ich bin Annika. Ich hatte 2015 in der Notunterkunft in Berlin, in der Rajaa und Namarek lebten, einen Minijob. Ich bin Hebamme und Heilerziehungspflegerin von Beruf. In der Notunterkunft war ich für den Bereich Partizipation und Frauenförderung angestellt. Ich arbeite aktuell in der Zukunftswerkstatt Heinersdorf gemeinsam mit Rajaa in der Kontakt- und Beratungsstelle „Hayatuna“.

Judith \& Salome: Wie war die erste Zeit in Deutschland? Wie und wo habt ihr gewohnt? 
Rajaa: Zunächst lebten wir in einer Turnhalle. Die ersten Jahre in Deutschland waren sehr schwer. So eng mit vielen Menschen zusammen zu leben, das Badezimmer mit 150 Personen zu teilen.

Namarek: Nachts durfte man nicht duschen und morgens warteten viele Leute. Man musste also früh aufstehen. Am besten war es morgens direkt nachdem das Reinigungspersonal sauber gemacht hat. Denn nach einer Stunde war es schon wieder schmutzig, da dort so viele Leute duschen müssen.

Rajaa: Manchmal gab es kranke Personen, die ansteckend waren. Sie haben uns Briefe gezeigt, dass sie ein eigenes Badezimmer benutzen müssen.

Annika: Ja, wir hatten ansteckende Krankheiten: Hepatitis oder Krätze. Wir haben alles gemeldet, aber keine Reaktion erhalten. Die Behörden waren zu diesem Zeitpunkt total überfordert.

Namarek: In der Sporthalle war es sehr schmutzig, ein Bett stand neben dem anderen. Es war ihnen egal, ob dort Männer, Frauen und Kinder direkt nebeneinander liegen. Keine Privatsphäre, überhaupt keine Privatsphäre.

Annika: Wir hatten am Anfang recht viele Bettwäschespenden und dann haben sich die Leute aus den Betten und Hochbetten Burgen gebaut. Dann haben wir eine Spende nichtbrennbarer Folie bekommen. Damit haben wir überall Kabinen gebaut, die nach oben hin offen waren. Das war eine Ehrenamtlichen-Aktion, das haben wir aus Spendengeldern bezahlt.

Zwei Tage danach hatten wir eine Begehung vom Lageso ${ }^{2}$, bei welcher sie sagten, dass wir das alles wieder abbauen müssen, das sei entgegen der Brandvorschriften. Aber wir haben das nicht gemacht. Wir haben gesagt, ok, dann reißt es ein, entzieht uns das Heim, aber das können wir den Leuten nicht wieder wegnehmen. Außerdem konnte man dort nichts einschließen, alles konnte geklaut werden. Die Menschen hatten noch kein Bankkonto, keine Arztscheine, noch gar nichts. Immer diese grünen Zettel, die ständig abgelaufen waren, die eine Woche gültig waren.

2 Landesamt für Gesundheit und Soziales, ab 2016 Landesamt für Flüchtlingsangelegenheiten (LAF), für die Unterbringung und Versorgung Flüchtender zuständige Stelle in Berlin. 
Rajaa: Ja, eine schlimme Situation. Das Essen war auch schrecklich. Du kannst dieses Essen nicht mal deinem Hund geben.

Namarek: Ich kenne deutsches Essen, von der Kita und der Schule meines Sohnes. Für uns ist das nicht perfekt, aber es ist ok und es ist gesund. Auch arabisches Essen mögen manche Menschen, andere nicht. Das ist normal, das ist kulturell unterschiedlich. Aber dieses Essen, war... ich weiß nicht.

Annika: Es war in Aluminium verpackt. Es wurde einmal am Tag geliefert. Das war auch nicht mehr warm und mehrmals war es verschimmelt. Man hat richtig gesehen, dass es nicht gut ist. Die Leute der Essensausgabe haben sich geschämt das Essen herauszugeben. Und die Menschen, die dort gelebt haben, mussten es essen.

Judith \& Salome: Was habt ihr dann gemacht?

Namarek: Wir sagten ihnen: Geben Sie uns das Geld, das Sie der Firma geben, und wir werden unser Essen kaufen oder wir werden draußen im Restaurant essen. Alles, nur etwas anderes als das. Aber es gibt keine Lösung. Wir haben es versucht und versucht. Wir mussten es sechs Monate lang essen, bis der Caterer gewechselt wurde.

Rajaa: Dann hatten wir die Idee selbst zu kochen. Aber sie haben gesagt, nein, das geht nicht.

Ein weiteres Problem war, dass das Frühstück um 8.30 Uhr oder um 8 Uhr kommt, die Kinder jedoch um 7 Uhr zur Schule müssen. Zurück kommen sie erst gegen 16 Uhr. Das Mittagessen kommt aber schon um 12.30 Uhr oder 13 Uhr und wird nach zwei Stunden weggeräumt. Die Kinder bekommen also weder Frühstück noch Mittagessen, lediglich das Abendessen, welches um 18 Uhr kommt.

Namarek: Die Unterbringung war so schlimm, dass wir manchmal an unserer Entscheidung zu fliehen zweifelten. Im Irak hatten wir Essen, wir haben Häuser, aber es ist nicht sicher. Aber hier haben wir kein Essen, wir haben kein Haus, wir haben keine Privatsphäre und wir wissen nicht, was los ist und was passieren wird. Aber jetzt ist es wirklich besser. Alles hat sich verändert.

Judith \& Salome: Wie war euer Verhältnis zu den Betreibenden und zur Heimleitung? 
Annika: Die Betreibenden standen hinter unserem Engagement und haben es auch nicht wirklich einfach gehabt mit unserer Beschwerdekultur. Wir haben das ja als Mitarbeiter:innen gesehen, was es dort für Essen gibt. Wir haben Fotos gemacht und uns immer wieder beschwert. Auch die Betreibenden haben sich dann beschwert, mit unseren Informationen.

Dann haben die Bewohner:innen die erste Unterschriftensammlung gemacht. Dann hat sich auch ein bisschen was verändert. Es gab einen neuen Caterer. Das Essen hat den Leuten zwar immer noch nicht geschmeckt, aber es war nicht mehr alt und nicht mehr verschimmelt. Wir versuchten die Möglichkeiten soweit es geht auszuloten. Das kam mit dem „lasst sie doch wenigstens selber kochen“. Da haben wir Kochstellen aufgestellt mit Kochplatten. Das war wieder so, die Betreibenden haben da einfach ein Stück weit drüber hinweggesehen, weil sie es verstehen konnten. Keine zwei Tage später, ähnlich wie bei diesen Abtrennungen, kam dann das Gesundheitsamt und sagte, das sei strikt verboten, Brandschutzvorrichtungen.

Wir haben es dann trotzdem gelassen. Als das Lageso wirklich mit der Schließungsdrohung kam, haben wir gesagt, okay dann stellen wir es wenigstens draußen hin. Dann hat sich jedoch die Schulleiterin von der Schule nebenan beschwert. Die Leute haben versucht, ihre eigenen Lösungen zu finden und das hat man wieder zerstört. Das war für mich das schlimmste.

Rajaa: Annika und andere Nachbar:innen haben uns in ihre Wohnungen eingeladen. Aber das ging nicht für 150 Personen und nicht jeden Tag. Ein anderes Problem waren die Security. Am Anfang haben wir die Regeln in Deutschland nicht verstanden. Ich dachte, die Security kann alles entscheiden, auch ob wir zurück in den Irak müssen. Wir hatten Angst vor ihnen. Und sie nutzten das aus, gegenüber Frauen und Kindern. Sie öffnen z.B. einfach die Badezimmertür. Wir beschwerten uns bei der Sozialberatung, ihr schien es jedoch egal zu sein, denn sie haben es immer wieder gemacht.

Annika: In der Turnhalle war die Security 24 Stunden am Tag vor Ort und die Sozialarbeiter nur von $8-22$ Uhr. Wir hatten als Mitarbeiter:innen immer ein schlechtes Gefühl, wenn wir die Bewohner:innen mit der Security allein gelassen haben. Ich und auch meine Kolleg:innen haben die Unterkunft oft mit einem schlechten Gefühl verlassen.

Wir haben gehört, dass Mitarbeiter der Security alleinstehenden Frauen die Hoffnung gemacht haben, gegen Sex einen Aufenthaltstitel oder eine Wohnung für sie zu organisieren. Obwohl wir uns so viel Mühe gegeben 
haben, dass das bei uns nicht passiert, habe ich so viele Geschichten gehört. Auch, dass Bewohner:innen zum Verkauf von Drogen angeworben wurden. Die Bewohner:innen waren so verzweifelt. Für mich ist bis heute die Frage, ob das Engagieren von Security überhaupt einen Vorteil hat, weil wir so viele negative Erfahrungen mit der Security gemacht haben. Manche waren besonders schlimm, die kamen zur Arbeit in einer Art Kampfmontur.

\section{Namarek: Dort stand Islamischer Staat darauf.}

Annika: Der einen Frau haben sie gesagt, sie darf keine Jeanshosen tragen. Die haben sich verhalten wie eine Schariapolizei. Wir haben uns immer dafür eingesetzt, dass wir Frauen im Team der Security brauchen. In den obersten Bereich, wo die Frauen duschen, sollte kein männlicher Security-Mitarbeiter gehen dürfen. Als wir diese Apartmentstruktur mit den abgehängten Tüchern hatten, gab es oben eine Galerie. Dann sind die Security immer einfach ständig oben auf die Galerie gegangen. Dann konnten die Frauen wieder nicht ihre Kopftücher absetzen, weil sie von oben reingucken konnten.

Judith \& Salome: Du hast gesagt, dass ihr euch dafür eingesetzt habt, dass bei der Security auch Frauen arbeiten. Hat das geklappt?

Annika: Ja, es war schwer, aber es hat geklappt, so ein bisschen. Wir hatten eigentlich in jeder Schicht eine Frau, außer in der Nachtschicht, das war schwierig. Und sobald wir mal nicht dran blieben, uns nicht immer wieder darüber beschwert haben, hat das dann auch nachgelassen. Man muss halt ständig dranbleiben. Wir hatten mit der Security wirklich wahnsinnig viel Streit. Ein Security hat einem Passanten, der betrunken vor dem Haus seinen Laptop verloren hatte, diesen geklaut. Ein Bewohner hat das mitbekommen und das dann gemeldet. Er wurde anschließend von dem Securitymitarbeiter verprügelt.

Rajaa: Die Security hat unsere Kabinen durchsucht. Bis wir es Annika erzählt haben, dachten wir, das sei erlaubt.

Annika: Die wenigen Rechte, die ein Mensch in so einer Situation hat, zu wahren, ist mir sehr wichtig gewesen. Deshalb habe ich alle motiviert uns alles zu erzählen, was in der Unterkunft passiert. Am Anfang war es total schwierig ihnen zu sagen, ihr müsst euch beschweren. Niemand spricht 
für euch, wenn ihr es nicht selbst tut. Wenn wir was verbessern wollen, dann müssen wir uns beschweren. Beschweren ist positiv.

\section{Umzug in eine Gemeinschaftsunterkunft}

Judith \& Salome: Wann konntet ihr aus der Turnhalle ausziehen und wie war eure Lebenssituation danach?

Rajaa: 2016 konnten wir endlich in ein anderes Heim, eine Gemeinschaftsunterkunft, umziehen. Die Unterkunft war für uns zunächst wie der Himmel.

Rajaa: In der neuen Unterkunft hatte jede Person Anspruch auf sieben Quadratmeter. Das ist schwierig, aber besser als die Sporthalle. Später kam es jedoch auch dort zu Problemen, aufgrund der verschiedenen Tagesrhythmen und Bedürfnisse der Personen, die sich ein Zimmer teilen. Manche kommen von der Schule und wollen schlafen, andere wollen Hausaufgaben machen. Einer muss schlafen und einer mit seiner Familie telefonieren.

Namarek: Ein Mann hatte Asthma, aber der Mann, mit dem er sich das Zimmer teilte, rauchte weiterhin darin.

Judith \& Salome: Wie viele Personen teilten sich dort ein Bad?

Rajaa: Es war wie eine Wohnung. Es gab zwei Zimmer. Davon war das $14 \mathrm{qm}$ große Zimmer für zwei Personen, das andere war $21 \mathrm{qm}$ groß und für drei Personen. Alle teilten sich ein Bad. Einer meiner Söhne ist 23 Jahre alt, der andere ist 15 Jahre alt. Meine Tochter ist 18 . Wenn sie ihre Kleidung wechseln wollte, musste sie ins Badezimmer gehen. Wir konnten auch niemanden einladen, da wir kaum Platz hatten.

Judith \& Salome: Wo war die Küche? Wie viele Personen teilten sie sich?

Namarek: Die Küche war im Flur.

Rajaa: 30 Personen teilten sich eine Küche, also fünf oder sechs Wohnungen teilten sich eine Küche.

Judith \& Salome: Wie war das für euch? 
Rajaa: Es gab Probleme mit der Küche. Wir mussten den Herd und die Küche selbst saubermachen. Wir haben das nicht verstanden, da es einen externen Putzdienst gab. Aber okay, ich habe es akzeptiert. Wenn ich dann am Morgen in die Küche kam und Frühstück machen wollte, war sie jedoch abgeschlossen, da die jungen Leute nachts dort gegessen hatten und die Unterkunft zur Strafe die Küche abgeschlossen hat.

Judith \& Salome: Wieso?

Rajaa: Weil die Küche schmutzig ist, hat die Security gesagt. Die Mitarbeiter:innen haben gesagt, wir sollen das untereinander klären, wir sollten einen Putzplan machen, in welchem eingeplant ist, wer wann putzt.

Annika: Sie sollten selbst verwalten, wer an welchem Tag putzt. Für die eine Küche hat das einer gemacht, weil er so verzweifelt war, dass die Küche ständig abgeschlossen war. Der hat dann diesen Putzplan quasi verwaltet. Er ist dann immer mit dem Handfeger und dem Putzzeug, das er auch noch von seinem Geld gekauft hat, zu den Leuten gegangen und hat gesagt, du hast heute Putzdienst. Deshalb gab es auch immer wieder Streit. Aber er hat es halt gemacht, weil sie vier Kinder hatten und die Küche immer zu war.

Namarek: Eine Frau kann nicht zu den Männern in die Zimmer gehen und ihnen sagen: „Sie sind heute an der Reihe mit dem Putzen.“ Die Männer würden sagen: „Wer bist du, um mir Befehle zu geben? Ich werde nicht putzen. Ich esse die ganze Zeit draußen.“ Das sagten sie immer wieder: „Wir essen hier nicht, wir essen draußen.“ Und wir wussten nicht, was wir tun sollen.

Rajaa: Wir sprachen dann mit den Mitarbeiter:innen, um eine Lösung zu finden. Leider ohne Erfolg.

Einmal, als Ramadan war, habe ich die ganze Küche geputzt. Dann musste ich zur Schule gehen. Vorher habe ich mit den Mitarbeiter:innen gesprochen und ihnen erklärt: „Diesen Herd habe ich geputzt, bitte schließt die Küche nicht ab.“ Ihre Antwort war: „Ja ok.“ Eigentlich hätte ich den Herd mit in meine Wohnung nehmen können, da wir als Familie 13 Personen sind. Aber ich habe das akzeptiert, dass die Mitarbeiter:innen uns das nicht erlaubten. Als ich in an dem Tag abends zurückkam, sah ich, dass die Küchentür abgeschlossen war. Erst sprach ich mit den Mitarbei- 
ter:innen darüber, dann rief ich den Chef. Nachdem wir gestritten haben, haben sie die Küche geöffnet.

\section{Wohnungssuche}

Judith \& Salome: Wie habt ihr eure Wohnungen gefunden?

Rajaa: Die Wohnungssuche war ein Problem. Das Jobcenter oder Sozialamt bezahlt bei Unterbringung in einem Heim $450 €$ pro Person. Das sind $2250 €$ bei fünf Personen. Aber als wir für uns eine Wohnung für $900 €$ oder $1100 €$ gefunden hatten, wollten sie das nicht bezahlen, es sei zu viel. Zudem hatten wir keine Erfahrung mit der Wohnungssuche in Deutschland, dass man das z.B. über eine Vermittlung machen kann. Ich denke, es sollte jemanden im Heim geben, der uns bei der Wohnungssuche unterstützt.

Namarek: Und für alle, ohne Rassismus. Es gab eine Mitarbeiterin in unserem Heim, ich sage ihren Namen nicht. Sie hat geholfen. Das habe ich selbst gesehen. Sie half einigen Menschen und sie fanden viele Wohnungen. Und als ich dorthin ging und ihr sagte, dass wir auch Unterstützung brauchen, sagte sie: „Füllen Sie dieses Papier aus, Sie können es selbst tun.“ Ich sagte ihr, dass sie mir helfen sollte, wie den anderen Leuten. Sie sagte: „Sie [die anderen Leute] können das nicht. Ich sehe, dass du studiert hast, Du kannst Lesen und Schreiben. Du kannst es selbst tun.“

Rajaa: Wenn wir die Bewerbungen in unserem Namen geschickt haben, bekamen wir manchmal keinen Besichtigungstermin, auf Annikas Bewerbungen haben wir mehr positive Rückmeldungen bekommen.

Rajaa: Ohne Hilfe hätten wir keine Wohnung gefunden.

Annika: Die Wohnung für Rajaa war ziemlich schwer zu finden, weil es eine 4-Raum-Wohnung sein musste. Das Problem ist ja auch, dass es diese Regeln bezüglich der Familiengröße und der notwendigen Wohnungsgröße gibt: Es ist kein Problem, dass die gesamte Familie auf 35 qm im Heim lebt. Aber es ist ein Problem, wenn sie auf $60 \mathrm{qm}$ in einer Wohnung lebt. Das schlimmste war, jemandem diese unsinnige Regel erklären zu müssen. Bis heute ist das so. Die Menschen aus der Gemeinschaftsunterkunft sagten damals: „Ja, Annika, aber es sind zwei Zimmer mehr, als wir im Heim 
haben. Wieso ist es zu wenig?“" „Ja, es ist nicht meine Regel,“ antworte ich dann. Ich verstehe ja die Problematik der Überbelegung von Wohnungen und dass es sinnvoll ist, eine angemessene Wohnraumgröße zu berücksichtigen, aber das kann ich niemandem erklären, der in so einer beengten Situation im Heim lebt. Für Rajaas Familie mussten wir anfangs sogar eine 5-Zimmerwohnung suchen, weil Mustafa noch dabei war. Genau so war es nämlich, wir mussten eine 5-Zimmerwohnung suchen.

Judith \& Salome: O Gott, in Berlin eine 5-Zimmerwohnung! Wie lange habt ihr gesucht?

Namarek: Von dem Moment an, als wir nach Deutschland kamen [lacht].

Rajaa: 2018 haben wir diese Wohnung bekommen und nach Deutschland sind wir 2015 gekommen.

Annika: Ich weiß es noch, dass wir irgendwann mal Rajaas Bewerbungen gezählt haben und das waren über 100, zu denen es mindestens eine Rückantwort oder Besichtigung gab.

Rajaa: Ich habe ganz Berlin gesehen. Einmal habe ich sechs Wohnungen an einem Tag besichtigt, bei minus $20 \mathrm{Grad}$.

Judith \& Salome: Wie habt ihr diese Wohnung gefunden?

Namarek: Annika hat sie gefunden. Ich wollte nicht einmal die Wohnungen sehen. Ich hätte überall alles akzeptiert. Ich wollte sie nur haben.

Annika: Ich suche die ganze Zeit auf einschlägigen Immobilienportalen. Ich arbeite nach und nach alle Wohnungsbewerbungen $\mathrm{ab}$, ein bisschen auch nach Dringlichkeit.

Rajaa: Als wir in diesem Beschwerdemanagement-Projekt gearbeitet haben, sollten wir nicht im Heim bleiben. Es würde nicht gehen, die Beschwerden aufzunehmen und im gleichen Heim zu wohnen.

Namarek: Wir haben in fünf Unterkünften Beschwerden aufgenommen. Wir müssen die Menschen nach den Problemen fragen und was los ist. Es war ein Pilotprojekt. Eine dieser Unterkünfte war unsere. Deshalb sagten sie, wir dürfen nicht an dem Ort arbeiten, an dem wir leben. Also versuchten sie einfach so schnell wie möglich eine Wohnung zu finden. 
Gründung der Monitoring Group und Entwicklung eines Konzepts für ein unabhängiges Beschwerdemanagement

Judith \& Salome: Das ist ein guter Übergang. Wir möchten gern ein wenig mehr über die Monitoring Group erfahren. Könnt ihr uns bitte erzählen, warum ihr sie gegründet habt?

Rajaa: Damals in der Turnhalle setzten wir uns zusammen, um nach Lösungen für die schwierige Unterkunftssituation zu suchen, wegen der Essenssituation und wegen der Security. Wir trafen uns jeden Freitag in einem sehr kleinen Zimmer in der Nähe des Badezimmers. Wir Frauen wollten etwas unternehmen, nach Lösungen suchen.

Judith \& Salome: Wie kam es, dass es nur Frauen waren?

Rajaa: Weil die Männer damals Angst hatten. Wir kannten damals die Regeln noch nicht und sie hatten Angst.

Namarek: Wir dachten, sogar die Security kann uns in unser Land zurückschicken. Wir wussten nicht, wer der Chef ist. Wir wussten nicht, wie das läuft. $\mathrm{Ob}$ sie uns in unser Land deportieren und wir alles verlieren werden, wenn wir etwas falsch machen. Also, die Männer, sie hatten wirklich Angst. Sie sagten immer wieder: Sag nichts, rede nicht, beschwere dich nicht einmal. Weil sie uns vielleicht zurückschicken. Wir hatten keinen Aufenthaltstitel. Wir warteten nur auf unser Schicksal.

Judith \& Salome: Was hat dein Ehemann gesagt? Ihr habt gesagt, dass es einigen Ehemännern nicht gefallen hat und dass die Männer Angst hatten. Hatte er keine Angst, dass ihr in Schwierigkeiten geraten könntet?

Namarek: Nein. Er hat für uns gekocht und sich um uns gekümmert, weil er Koch ist, das ist seine Arbeit und er wollte mich auch unterstützen.

Judith \& Salome: Was haben die anderen Männer gesagt?

Namarek: Sie mussten es akzeptieren. Wenn er nein sagen würde, würde sich seine Frau vielleicht streiten und sich scheiden lassen. Es ist in Deutschland nicht wie in unserem Land. Dort würde sie an ihre Kinder denken. Die Kinder würden beim Vater bleiben. Also würde sie alles verlieren. Und jeder würde sagen, sie ist eine geschiedene Frau, niemand würde sie heiraten, weil sie geschieden ist. Sie würden sagen, es ist ihre 
Schuld. Sie würden denken, vielleicht stimmt etwas mit ihr nicht, dass er sich von ihr scheiden ließ und sie verließ. Aber hier ist es anders, wenn die Männer die Frauen verlassen haben.

Annika: Ich hatte das Gefühl, dass es hilfreich war, dass wir auch manchmal Probleme gelöst haben. Dass die Männer auch froh waren, weil die Frauen eigentlich die ganze Arbeit übernommen haben. Also, wir haben neben dem, dass wir uns beschwert haben, auch für Verbesserungen gesorgt. Wir haben Wohnungen gesucht oder Papierkram erledigt und so waren sie auch froh, weil wir viel erledigt haben. Wir haben uns zwar getroffen und über Beschwerdemanagement gesprochen, aber wir haben auch immer Papiere angeguckt oder Lösungen überlegt, wenn jemand ein akutes Problem hatte.

Judith \& Salome: Hattet ihr Angst, euren Aufenthaltstitel zu verlieren?

Namarek: Ja, natürlich. Es war wirklich gefährlich auf dem Wasser und all das, um hierher zu kommen. Und wir sind nicht bereit, unseren Platz hier zu verlieren. Weil es unser Zuhause ist, es ist unser Leben in Deutschland. Es gibt für uns kein anderes Land, wir kämpfen für unser gutes Leben in diesem Land.

Rajaa: Wir haben uns entschieden zu sprechen, nur wir Frauen. Nicht alle Frauen, manche hatten Angst. Annika hat einmal eine Anwältin eingeladen oder jemand von der Sozialberatung. Sie haben uns erzählt, was unsere Rechte sind und was wir machen können. Endlich verstanden wir unsere Situation etwas besser. Wir suchten nach Lösungen für unsere Situation. Annika hat den Kontakt zu den Leuten hergestellt. Sie als Deutsche schrieb die Mails und kommunizierte mit dem Senat und anderen.

Annika: Unser erstes, großes Treffen mit Berliner Behörden war Anfang 2017 mit dem Staatssekretär für Integration, also der Senatsverwaltung. Wir haben ihn nach Heinersdorf eingeladen und er ist gekommen. Wir hatten uns gedacht, die Frauen müssen einfach nochmal sagen, was ihre Probleme sind. Teilgenommen haben Frauen aus Eritrea, Moldawien, Afghanistan, dem Irak und Syrien, also aus allen möglichen verschiedenen Ländern. Wir hatten es so gemacht, dass die eine die Geschichte von der anderen erzählt. Also, dass nicht du selbst dein Problem beschreibt, sondern jemand anderes. Die Geschichten, die wirklich auch alle erlebt hatten. Der Staatssekretär hat bei dieser ersten Veranstaltung gedacht, er 
kommt für eine halbe Stunde. Dann ist er da reingekommen, ein voller Raum, so viele Frauen. Wir waren glaube ich 40 Frauen.

Dann wurden wir eingeladen, um am Partizipationsprozess zum neuen Gesamtkonzept zur Integration und Partizipation Geflüchteter des Beauftragten des Berliner Senats für Integration und Migration teilzunehmen. Und wieder ausgeladen. Aber dann sind wir trotzdem immer alle gekommen. Dann war der große Workshop im Wannseeforum. Voll schön, das war echt wie Urlaub. Dann hatten wir Projektgelder bekommen und dann haben wir dieses Policypaper geschrieben, mit der Unterstützung von zwei Lektorinnen. Ein halbes Jahr danach hat das Pilotprojekt „Unabhängiges Beschwerdemanagement für geflüchtete Menschen in Unterkünften“ gestartet.

Namarek: Wir waren am Anfang sehr hoffnungsvoll. Wir sind in die verschiedenen Unterkünfte gegangen und haben den Bewohner:innen gesagt, dass wir kommen, um ihnen bei ihren Beschwerden zu helfen. Aber sie sagten immer wieder: „Nichts wird sich ändern, wir haben schon viel geredet. Viele haben mit uns geredet, aber nichts hat sich geändert. Wir wollen nicht noch einmal reden." Und wir von dem Pilotprojekt mussten ihnen sagen: „Nein, wir haben etwas anderes und wir sind nicht wie die anderen."

Schließlich begannen sie uns zu vertrauen. Wir haben alle Beschwerden aufgeschrieben, aber es gab keine Lösungen für die meisten Probleme. Wir sind dann immer öfter im Büro geblieben, weil wir den Menschen, die Hoffnung auf Verbesserung hatten und denen wir Unterstützung versprochen haben, nicht mehr ins Gesicht sehen konnten.

Annika: Namarek und Rajaa haben mitgearbeitet, die Beschwerden aufzunehmen und ich war in der Steuerungsgruppe. Unser Konzept am Anfang war, dass wir gesagt haben, dass Menschen die Beschwerden aufnehmen sollten, die selbst in einem Heim gelebt haben, denn dann hat man auch einen Blick für die Probleme dort. Du hast die Erfahrung und du hast das Vertrauen. Aber die Probleme können nicht sie lösen, sondern die muss die Ebene darüber lösen. Das ist aber nicht passiert. Die Vertrauenspersonen, die Probleme aufgenommen haben, haben sich dann ausnahmslos geschämt, den Leuten wieder unter die Augen zu treten, weil es keine Lösung gab.

Die Beschwerden sind stark zurückgegangen, weil die Beschwerdeführenden auch gemerkt haben, dass es keine Lösungen gibt. 


\section{Aktivitäten der Zukunftswerkstatt Heinersdorf}

Judith \& Salome: Wie arbeitet ihr jetzt, in der Zukunftswerkstatt? Wie löst ihr dort die Probleme?

Rajaa: Die Frauen kommen zu mir und erzählen ihre Probleme. Als Team überlegen wir uns immer gemeinsam mit den Menschen, welche Lösungen es gibt. Und diesen Weg gehen wir dann gemeinsam.

Annika: Rajaa und Namarek haben viel Kontakt zu Frauen, aus Unterkünften, aus Friseursalons, vom Einkaufen, aber auch über Facebookgruppen.

Judith \& Salome: Könnt ihr von einem Beispiel erzählen?

Rajaa: Zum Beispiel eine Frau aus einer Sammelunterkunft. Sie ist sehr krank und sie wohnt in einem Heim. Sie hat keine sozialen Kontakte. Sie ist auf der Suche nach Arbeit und nach einem Deutschkurs. Nachdem sie uns von ihrer Situation berichtet hatte, hat Annika ihr einen Kurs gesucht und ihre Unterlagen bezüglich ihrer gesundheitlichen Situation sortiert sowie für die Wohnungssuche, z.B. für einen Wohnberechtigungsschein. Sie hat dann mit einem Deutschkurs begonnen und wir konnten eine Wohnung für sie finden. Auch hat sie eine kleine Arbeit bei uns, einmal die Woche. Wir suchen derzeit einen Minijob für sie.

Ein anderes Beispiel ist eine Frau, die hatte in ihrer Heimat studiert und gearbeitet. Aber hier konnte sie nichts machen. Sie hatte Angst vor ihrem Mann, er war gewalttätig ihr gegenüber. Aber als sie Kontakt mit uns aufgenommen hat, wurde sie stärker und selbstbewusster. Sie ist sehr stark geworden und fing an zu arbeiten und hat ihm gesagt, dass sie ihn verlässt, wenn er sich nicht ändert.

Unterbringung und Unterstützungsmöglichkeiten während der Covid-19Pandemie

Judith \& Salome: Wie stellen sich Unterbringung und Unterstützung in Zeiten von Corona dar?

Rajaa: Wir unterstützen jetzt über WhatsApp.

Annika: Voll datensicher... [ironisch]. 
Rajaa: Für die Frauen ist das easy.

Annika: Bei vielen Sachen war ich erstaunt. So hatte jemand eine Androhung einer Zwangsvollstreckung von einem Mobilfunkanbieter erhalten. Normalerweise sind sie immer sehr streng, wenn ich für die Frauen dort anrufe, und sie sagen: „Nein, wir reden nur mit der betreffenden Person, nicht mit Ihnen." Doch in der Coronazeit hatte ich den Eindruck, dass viele Unternehmen den Datenschutz nicht so ernst genommen haben. Die Mitarbeiterin von einer Telefongesellschaft hat auf meine Anfrage geantwortet: „Sie wollen ihr ja helfen. Einen Vertrag in ihrem Namen würde ich mit Ihnen jetzt nicht abschließen. Aber wenn es hier um die Klärung von einem Sachverhalt geht, ist es ok."

Es gab die Leute, die untergetaucht sind und schwarzgearbeitet haben. Die haben zum Teil schon Jahre lang mit vielen Leuten in einer Wohnung gelebt, aber in der Pandemie haben plötzlich die Nachbar:innen gesagt: „Da steht nur einer an der Klingel und da wohnen aber fünf.“ Die Nachbar:innen haben sie dann angezeigt. Daraufhin ist zum Teil die Polizei gekommen. Sie haben deshalb ihre Schwarzarbeit verloren, also ihr Einkommen und ihre Schlafplätze. Und so kamen auch während der Pandemie Menschen, die davor geheim gelebt haben. Auch, weil sie gehört haben, dass jetzt die Möglichkeit besteht, eine Krankenversicherung zu bekommen, weil ja gerade die Abschiebungen nicht stattfinden. Da hatten wir auch ein paar Fälle in der Beratung.

Judith \& Salome: Habt ihr etwas aus den Heimen gehört? Wie ist dort die Situation?

Annika: Bis jetzt besteht ein Besuchsverbot. Das ist für viele sehr schlimm, dass Treffen in den Heimen gar nicht möglich sind. Zurecht haben die Betreibenden Angst, dass sich Corona in der Unterkunft ausbreitet. Massenunterkünfte sind stark gefährdet, viele Menschen auf engem Raum, gemeinsame Küchen und Sanitärräume. Zudem gibt es viele Menschen, die in die Kategorie „Risikopatient“ gehören. Es gab am Anfang keine Entzerrungsstruktur. Ich glaube, das hat sich jetzt ein bisschen verbessert.

Judith \& Salome: Wir bedanken uns bei euch für das Gespräch. Es tut uns leid, wenn dadurch wieder schlechte Erinnerungen aufgewühlt wurden.

Rajaa: Wir freuen uns über Eure Arbeit, wir wollen den Menschen helfen und hoffen, dass wir einen guten Beitrag leisten konnten. 
Namarek: Ich hoffe, dass wir andere Menschen, die ihre Rechte nicht kennen, unterstützen können. Sie müssen wissen, dass es sich lohnt, für sein Recht zu kämpfen. 